\title{
The Quality of the Early Motor Repertoire in Preterm Infants Predicts Minor Neurologic Dysfunction at School Age
}

\author{
Janneke L. M. Bruggink, MD, Christa Einspieler, PhD, Phillipa R. Butcher, PhD, Koenraad N. J. A. Van Braeckel, MSc, \\ Heinz F. R. PReChtL, DM, DPhil, and ARend F. Bos, MD, PhD
}

Objective The quality of a child's motor repertoire at age 3 to 4 months postterm is predictive of later cerebral palsy (CP). Its predictive power for minor neurologic dysfunction (MND) is unclear. This study aimed to investigate the predictive value of the quality of the early motor repertoire for the development of MND at school age.

Study design We assessed the motor repertoire from video recordings made at 6 to 24 weeks postterm in 82 preterm infants (mean gestational age, $29.7 \pm 1.9$ weeks; mean birth weight, $1183 \pm 302 \mathrm{~g}$ ). At age 7 to 11 years, Touwen's neurologic examination was performed, and the children were classified as normal $(n=49 ; 60 \%)$, MND $(n=18 ; 22 \%)$, or $\mathrm{CP}(\mathrm{n}=15 ; 18 \%)$.

Results Multiple logistic regression analysis showed that the quality of fidgety movements (FMs) and the quality of the concurrent motor repertoire had independent prognostic value for MND at school age. Abnormal FMs evolved into MND in $64 \%$ of the children. Nine of the 28 children with normal FMs and an abnormal concurrent motor repertoire developed abnormally (32\%). Only 1 child of the 21 children with normal FMs and a normal concurrent motor repertoire developed MND (5\%).

Conclusions Assessment of the quality of the early motor repertoire can accurately identify individual infants at high and low risk for MND at school age. (J Pediatr 2008;153:32-9)

$\mathbf{N}$ eurologic and developmental complications are common in infants born preterm. Well-documented sequelae of preterm birth that persist into childhood and adolescence include motor, cognitive, and behavioral impairments ranging in severity from cerebral palsy (CP) and sensory loss to minor neurologic dysfunction (MND), learning disabilities, and attention and behavior problems. ${ }^{1,2}$ The early identification of infants at highest risk remains difficult. In the last 15 years, the quality of spontaneous general movements (GMs), assessed following Prechtl's method, has emerged as a reliable and valid predictor of severe neurologic deficits (eg, CP) for the individual infant. ${ }^{3}$ This method is based on a visual Gestalt perception of the quality of GMs in the preterm, term, and postterm periods, up to 5 months postterm. The quality of so-called "fidgety movements" (FMs), which emerge at 6 to 9 weeks postterm and can be observed up to 20 weeks postterm, is a particularly accurate marker for neurologic outcome. Most infants (96\%) with normal FMs have normal neurologic outcomes, whereas most infants (95\%) in whom FMs are absent during this particular age period develop CP. ${ }^{4,5}$ An early indicator with comparable prognostic value for the milder deficits (eg, MND), which are far more prevalent than $\mathrm{CP}$ in this population, has not yet been identified.

Previously, associations have been found between the development of MND in childhood and the quality of GMs at 2 to 4 months postterm. ${ }^{6,7}$ Groen et $\mathrm{al}^{8}$ showed that abnormal GMs at 8 to 17 weeks postterm were associated with coordination problems and fine manipulative disability at school age; however, the predictive value of GMs for the individual infant was low. The abnormality of the GMs was based on the extent to which the complexity and variation of spontaneous movements were reduced. Abnormal FMs (particularly their absence), despite their high predictive value for $\mathrm{CP},{ }^{4}$ were not taken into account. In an earlier study, we used a different approach, carrying out separate global assessments of different qualitative aspects of the motor repertoire. ${ }^{9}$ We found that both an abnormal quality of FMs and a monotonous character of concurrent movements increased the probability of

\begin{tabular}{llll}
\hline Cl & Confidence interval & LR & Likelihood ratio \\
CP & Cerebral palsy & MND & Minor neurologic dysfunction \\
FM & Fidgety movement & NICU & Neonatal intensive care unit \\
GM & General movement & PVL & Periventricular leukomalacia \\
\hline
\end{tabular}

From the Department of Pediatrics, Division of Neonatology, Beatrix Children's Hospital, University Medical Center Groningen, Groningen, the Netherlands (J.B., A.B.); Institute of Physiology, Developmental Physiology and Developmental Neurology, Center for Physiological Medicine, Medical University, Graz, Austria (C.E., H.P.); and Department of Psychology, University Medical Center Groningen, Groningen, the Netherlands (P.B., K.vB.).

Submitted for publication Aug 17, 2007; last revision received Nov 26, 2007; accepted Dec 19, 2007

Reprint requests: Janneke L. M. Bruggink MD, Department of Pediatrics, Division of Neonatology, Beatrix Children's Hospital, University Medical Center Groningen, University of Groningen, Hanzeplein I, PO Box 30.001, 9700 RB Groningen, The Netherlands. E-mail: j.l.m.bruggink@bkk.umcg.nl. 0022-3476/\$ - see front matter

Copyright (C) 2008 Mosby Inc. All rights reserved.

10.10 | 6/j.jpeds.2007.12.047 
complex MND

Children who developed CP

\begin{tabular}{|c|c|}
\hline Number & 49 \\
\hline Gestational age, weeks & 30.1 (28.6 to 31.7$)$ \\
\hline Birth weight, $g$ & 1160 (950 to 1343$)$ \\
\hline Male infants & $23(47)$ \\
\hline IUGR (birth weight $<5$ th percentile) $\dagger$ & $12(24)$ \\
\hline Prenatal corticosteroid use & 34 (7I) \\
\hline Apgar score at 5 minutes & $8(8$ to 9$)$ \\
\hline Umbilical pH & 7.28 (7.25 to 7.31$)$ \\
\hline Ventilator support (IPPV of HFOV) & $23(47)$ \\
\hline Septicemia & $17(35)$ \\
\hline $\mathrm{ICH}$ grade I to $2 \pi$ & II (22) \\
\hline $\mathrm{ICH}$ grade 3 to 4 & None \\
\hline PVL grade It† & $19(39)$ \\
\hline PVL grade 2 to 3 & None \\
\hline BPD㧊 & II (22) \\
\hline Postnatal corticosteroid use & $3(6)$ \\
\hline
\end{tabular}

18

$28.9(27.8$ to 31.0$)$

$1165(898$ to 1333$)$

$12(67)$

$4(22)$

II (6I)

$8(5$ to 8.3$) \ddagger$

$7.26(7.21$ to 7.33$)$

$11(61)$

$7(39)$

$6(33)$

None

$14(78) \ddagger$

None

$5(28)$

$4(22)$
15

28.7 (27.7 to 30.0$)^{*}$

1220 (870 to 1460$)$

$12(80)$

I (7)

$9(60)$

$6(5$ to 7$) \ddagger$

$7.26(7.21$ to 7.33$)$

$15(100) \ddagger, \S$

5 (33)

2 (13)

$5(33) \ddagger$,**

8 (53)

$4(27) \ddagger$,**

$9(60) \ddagger$

$9(60) \ddagger$,**

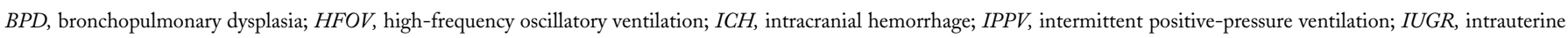
growth restriction.

Data are expressed as median (25th to 75 th percentile) or as $n$ (\%).

$* P<.05$ compared with infants who developed normally or simple MND.

$\dagger$ Birth weight according to the Dutch weight centiles of Kloosterman. ${ }^{22}$

$\ddagger P<.01$ compared with infants who developed normally or simple MND.

$\S P<.01$ compared with infants who developed complex MND.

ПGraded according to Papile et al. ${ }^{23}$

${ }^{* *} P<.05$ compared with infants who developed complex MND.

††Graded according to de Vries et al. ${ }^{24}$ PVL grade 1 is also called prolonged flaring.

$\ddagger \ddagger$ Defined as oxygen dependency at 36 weeks postmenstrual age.

developing MND at age 2 years., ${ }^{3,9}$ The question then arises as to whether the assessment of the quality of FMs, in conjunction with the assessment of the quality of the concurrent motor repertoire, also has predictive value for MND at school age. Because FMs emerge at 6 weeks and disappear at around 20 weeks postterm, we investigated whether the quality of the motor repertoire at 6 to 24 weeks postterm has prognostic value for MND at age 7 to 11 years in preterm infants.

\section{METHODS}

\section{Subjects}

The study group comprised 82 infants $(50$ boys and 32 girls) born preterm between September 1992 and October 1997 and admitted to the neonatal intensive care unit (NICU) of the Beatrix Children's Hospital of the University Medical Center of Groningen. The infants were members of a larger group of 99 infants who were included in prospective studies of the prognostic value of the quality of GMs for neurologic and developmental findings. The results of these studies have been reported previously., ${ }^{5,10-15}$ The study group can be considered a representative sample of the preterm infant population in our NICU (tertiary referral center) during the mid1990s.

Seven infants died during the first few months of life, most due to severe respiratory problems. Conditions that could interfere with normal neurologic development became apparent in 3 infants ( 2 with blindness due to retinopathy of prematurity and 1 with morbus Duchenne). Five of the remaining 89 infants could not be traced. Two families refused to participate. Obstetrical and neonatal data are listed in Table I, grouped according to the child's neurologic status at school age. All parents gave written, informed consent. The University Medical Center of Groningen's Ethical Review Board approved the study design.

\section{Recording and Evaluation of the Motor Repertoire between 6 and 24 Weeks Postterm}

Video recordings, approximately 10 minutes long, were made of the infants at approximately 6 to 8,12 to 14 , and 18 to 21 weeks postterm. The timing and frequency of the video recordings differed for a few infants for logistical or family reasons. The recordings were made at either the outpatient clinic or home during periods of active wakefulness between feeds, with the partly dressed infant lying in the supine position.

A total of 214 recordings (median, 3 per infant; mean duration, 9:01 minutes) were available for analysis. The recordings of all infants were ordered according to increasing postterm age and evaluated offline by 3 of the authors (J.B., A.B., and C.E.) according to the criteria of Einspieler et al. ${ }^{3}$ Two of the evaluators were unaware of the infant's clinical history and neurologic status; 1 evaluator knew the infant's clinical history. When the evaluators disagreed on the characterization of an infant, all 3 reevaluated that infant's record- 
ings; in all cases, consensus was reached on a final score. The quality of the motor repertoire could not be judged in 10 of the 214 recordings (4.7\%), due to infant crying, sleepiness, or hiccups.

We assessed the quality of FMs and the quality of the concurrent motor repertoire separately during different runs of the videotapes. For further analysis, we clustered the video recordings at age 6 to 10 weeks postterm (early FM period), 11 to 16 weeks (mid-FM period), and 17 to 24 weeks (late or post-FM period). If 2 recordings were made of the same infant during the same age period, then the recording closest to the median age of the particular age period was selected. We chose these periods because of the age-related characteristics of the quality of FMs and also because over time, the number of movement patterns, and thus also the complexity of the motor repertoire, increase considerably.,16

\section{Quality of FMs}

FMs are of small amplitude, moderate speed, and variable acceleration and occur in the neck, trunk, and limbs in all directions. They are continual in the awake infant, except during fussing and crying. ${ }^{4}$ FMs may be seen as early as 6 weeks postterm but usually appear around 9 weeks and persist until 15 to 20 weeks. ${ }^{3}$ We assessed the quality of FMs as normal, abnormal (exaggerated amplitude, speed, and jerkiness), or absent (no FMs observed between 6 and 20 weeks postterm). When FMs were present, their temporal organization was scored as continual $(++)$, intermittent $(+)$, or sporadic $(+/-),{ }^{17}$ and their spatial organization was scored as proximal (more prominent in the trunk, neck, shoulders, and hips), distal (more prominent in the wrists and ankles), or equally prominent in the proximal and distal parts of the body. ${ }^{3}$

\section{Quality of the Concurrent Motor Repertoire}

The quality of the concurrent motor repertoire was considered normal if it was smooth, variable, fluent, and complex. Reduced complexity (monotony), jerkiness, and/or stiffness were considered signs of abnormality and were scored separately. ${ }^{8,11}$ Differences in degree of monotony, jerkiness, and stiffness were not scored.

\section{Interscorer Reliability}

To check interobserver reliability, 145 randomly selected recordings were assessed by 3 observers. Cohen's $\kappa$ was 0.87 for the quality of FMs and 0.91 for the quality of the concurrent motor repertoire.

\section{Assessment of Neurologic and Motor Findings at Age 7 to 11 Years}

Follow-up consisting of pediatric and neurologic examinations was performed at regular intervals. At age 6 years, 15 children were diagnosed with $\mathrm{CP}$ according to Hagberg's criteria. ${ }^{18}$ A neurologic examination according to Touwen, ${ }^{19}$ designed to detect signs of minor neurologic dysfunction, was performed between age 7 and age 11 on the remaining 67 children. Following Hadders-Algra, ${ }^{20} 6$ subcategories of function were assessed: posture and muscle tone, reflexes, choreiform dyskinesia, coordination and balance, fine manipulative ability, and rare dysfunctions, including excessive associated movements. The children were classified as neurologically normal, simple MND, or complex MND. Simple MND denoted the presence of dysfunction in 1 or 2 subcategories; complex MND, the presence of dysfunction in more than 2 subcategories. ${ }^{6,20}$ Because simple MND has limited clinical and functional significance compared with complex MND, ${ }^{8,20}$ the normal and simple MND groups were analyzed as a single group. Furthermore, because the aim of the study was to evaluate the significance of the quality of the movement repertoire for minor neurologic deficits, the analysis was performed both with and without the children with CP.

The Movement ABC, a test of motor skill for children age 4 to 12 years, ${ }^{21}$ also was administered. This test, which is widely used in practice and in research, yields a score for total movement quality based on separate scores for fine motor skills, ball skills, and balance. The tasks composing the Movement $\mathrm{ABC}$ are representative of the motor skills that are required of children attending elementary school and are adapted to the child's age.

\section{Statistical Analysis}

Statistical analysis was performed using SPSS for Windows, version 14.0 (SPSS Inc, Chicago, IL). Fisher's exact test and the $\chi^{2}$ test for trend were used to evaluate the associations between the categorical measures of the quality of FMs and the concurrent motor repertoire on the one hand, and neurologic findings at school age on the other hand. The Kruskal-Wallis test and the Mann-Whitney $U$ test were applied to evaluate the associations between clinical data and later neurologic findings. To assess the influence of the different clinical and movement quality-related factors on later neurologic findings, backward multiple logistic regression analysis was performed. Only factors that were significant in the univariate analyses were included in the model. Throughout the analyses, $P<.05$ (2-tailed test) was considered statistically significant.

\section{RESULTS}

\section{Neurologic and Movement ABC Findings at School Age}

At age 7 to 11 years, 15 children had developed CP (5 unilateral, 8 bilateral, 2 dyskinetic). Of the remaining 67 children, 36 were neurologically normal, 13 had simple MND, and 18 had complex MND. Most children with complex MND had abnormalities in the clusters coordination/ balance and fine manipulative ability. This combination of abnormal clusters was present in only 3 of the children with simple MND.

All clusters of the neurologic test except reflexes correlated significantly with the Movement ABC scores. The total 
Table II. Association between quality of GMs at postterm age 6 to 10 weeks, II to 16 weeks, and 17 to 24 weeks and neurologic findings at school age

\begin{tabular}{|c|c|c|c|c|c|}
\hline \multirow[b]{2}{*}{ Postterm age, weeks } & \multirow[b]{2}{*}{ Quality of FMs } & \multicolumn{3}{|c|}{ Neurologic findings at school age } & \multirow[b]{2}{*}{ Total } \\
\hline & & Normal/simple MND & Complex MND & Cerebral palsy & \\
\hline \multirow[t]{3}{*}{6 to 10} & Normal & 30 & 7 & & 37 \\
\hline & Absent & 2 & 6 & II & 19 \\
\hline & Total & 34 & 15 & II & 60 \\
\hline \multirow[t]{3}{*}{11 to 16} & Normal & 39 & 9 & I & 49 \\
\hline & Absent & & 1 & 12 & 13 \\
\hline & Total & 43 & 17 & 13 & 73 \\
\hline \multirow[t]{4}{*}{17 to 24} & Normal & 21 & 4 & I & 26 \\
\hline & Abnormal & I & 2 & & 3 \\
\hline & Absent & 12 & 4 & 8 & 24 \\
\hline & Total & 34 & 10 & 9 & 53 \\
\hline
\end{tabular}

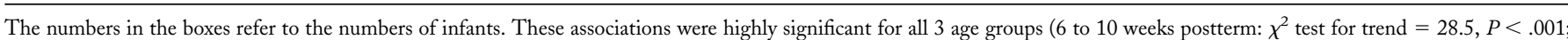
11 to 16 weeks postterm: $\chi^{2}$ test for trend $=62.4 ; P<.001 ; 17$ to 24 weeks postterm: $\chi^{2}$ test for trend $=7.6 ; P=0.011$ ).

score and all subtest scores on the Movement $\mathrm{ABC}$ were similar for the children with normal MND and those with simple MND. The total score and the subtest scores for fine motor and ball skills differed significantly between the children with simple MND and those with complex MND (Mann Whitney $U$ test, $P=.002, .022$, and .018, respectively). The difference in scores for balance almost reached statistical significance $(P=.056)$. These findings confirm the proposed differences in functional consequences between simple and complex MND and support the decision to combine the normal and simple MND groups for further analysis. (For the purpose of clarity, this combined group is referred to as "normal" in the rest of the article.)

\section{Relationship between Clinical Data at Birth and Neurologic Findings at School Age}

The associations between the perinatal and neonatal clinical data and the neurologic findings at school age are shown in Table I. Several clinical variables differed significantly between infants with $\mathrm{CP}$ and those who developed complex MND or were classified as normal. Hardly any differences existed between infants who developed complex MND and those classified as normal. Only a lower Apgar score at 5 minutes and the presence of prolonged flaring (periventricular leukomalacia [PVL] grade 1) were more prevalent in the MND infants.

\section{Relationship between Qualitative Aspects of the Motor Repertoire and Neurologic Findings at School Age} QuAlity of FMs Between 6 AND 24 WEeKs POSTTERM. The absence of FMs at 11 to 16 weeks postterm was most strongly associated with adverse neurologic outcome, preceding $\mathrm{CP}$ in 12 of 13 infants (92\%), preceding complex MND in 1 infant, and not being observed in any infant later classified as normal (Table II). Absence of FMs at 6 to 10 weeks and at 17 to 24 weeks was observed in several infants later classified as normal, however. In all of these infants, FMs were present at 11 to 16 weeks; they were normal in some infants and abnormal in others.

Abnormal FMs were observed most frequently at 11 to 16 weeks postterm. They preceded complex MND in 7 of 11 cases (64\%). The remaining 4 infants (36\%) were later classified as normal. Abnormal FMs were rare at both 6 to 10 weeks and 17 to 24 weeks. However, all but 1 of the infants who exhibited abnormal FMs at 11 to 16 weeks had either absent or abnormal FMs both before (6 to 10 weeks) and after (17 to 24 weeks) that period. Only 1 infant was inconsistent, demonstrating normal FMs at 6 to 10 weeks.

At any age, approximately $80 \%$ of the infants who exhibited normal FMs were classified as normal at school age. Normal FMs preceded CP in only 1 infant. Again, the association between normal FMs and normal neurologic outcome was strongest at 11 to 16 weeks. However, normal FMs at 11 to 16 weeks preceded complex MND in 9 of 49 infants (18\%).

Overall, the quality of FMs at 11 to 16 weeks had the strongest association with neurologic outcome. However, although it differentiated the infants with complex MND from the infants with $\mathrm{CP}$ very well, it differentiated the infants with complex MND from the normal infants less clearly. Only roughly half of the infants who developed complex MND (8 of 17) had abnormal FMs at 11 to 16 weeks; the other half ( 9 of 17) had normal FMs. The 2 groups did not differ in terms of the detailed aspects of the FMs, such as temporal characteristics and proximal distal predominance.

QUALITY OF THE CONCURRENT MOTOR REPERTOIRE BETWEEN 6 AND 24 WEEKS POSTTERM. At all ages, the concurrent motor repertoire was frequently abnormal, ranging from $68 \%$ to $72 \%$ of all infants. The association between the quality of the concurrent motor repertoire and neurologic findings was 

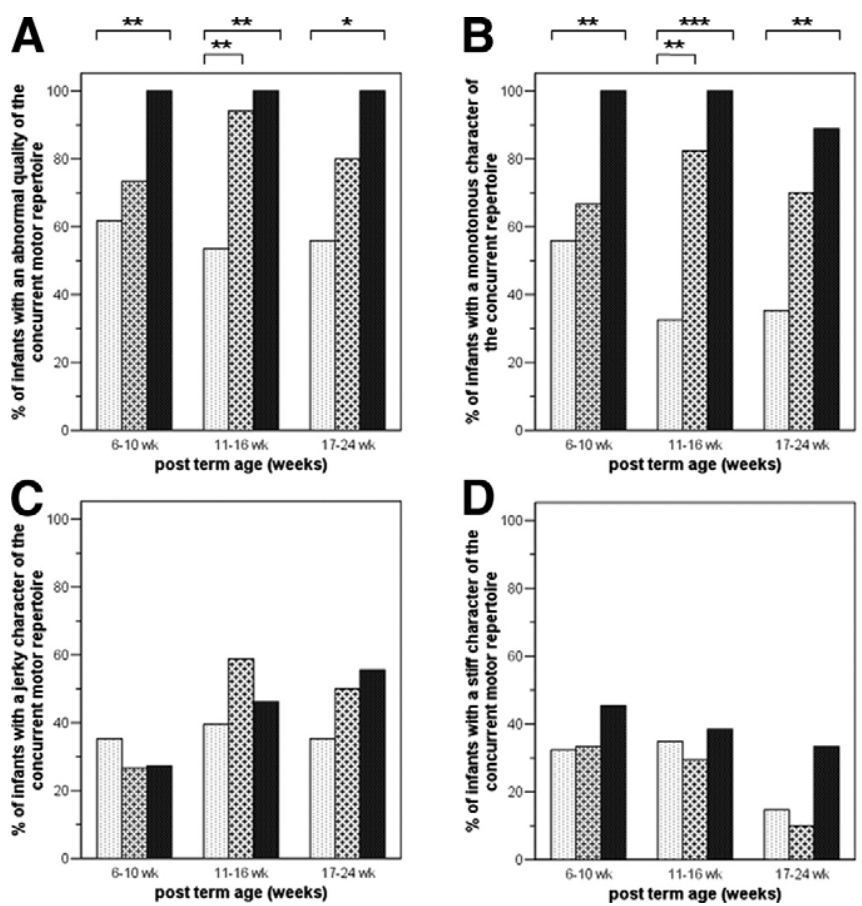

Figure. Relationship between the quality of the concurrent motor repertoire and neurologic findings at school age. For the groups of infants classified as either normal, complex MND, or CP at school age, the bars represent the percentage of infants with an abnormal quality of the concurrent motor repertoire $(\mathrm{A})$, in particular a monotonous character (B), a jerky character (C) or a stiff character (D). Differences between the groups were analyzed using Fisher exact test $\left({ }^{*} P<.05\right.$; ${ }^{* *} P<.01$; ${ }^{* * *} P<$ .001). Neurological findings at school age: Normal/Simple MND,

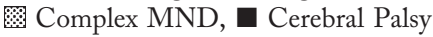

strongest at 11 to 16 weeks postterm. A normal concurrent motor repertoire at this age distinguished the infants who were later classified as normal from those who developed either complex MND or CP (Figure, A), preceding complex MND in only 1 of 21 infants (5\%) and never preceding CP (Table III; available at www.jpeds.com). An abnormal concurrent motor repertoire had less predictive value, however, preceding complex MND in $16(31 \%)$ and $\mathrm{CP}$ in 13 of 52 infants (25\%). Thus, nearly half of the infants (23 of 52; 44\%) whose concurrent motor repertoire was abnormal at 11 to 16 weeks were later classified as normal.

Three types of concurrent motor repertoire abnormality were scored: monotony, jerkiness, and stiffness. Of these, only monotony was associated with later complex MND (Figure, B). The concurrent motor repertoire was monotonous in $80 \%$ of infants who later developed complex MND, as opposed to $30 \%$ of infants later classified as normal. Jerkiness and stiffness at any age did not differentiate between the 2 groups (Figure, C, D).

\section{Prognostic Value of Combining Several Qualitative Characteristics of the Motor Repertoire for Later Complex MND}

At 11 to 16 weeks postterm, 2 aspects of the motor repertoire had particularly high prognostic value for the de- velopment of complex MND: the quality of FMs and the quality of the concurrent motor repertoire. The prognostic value of these 2 characteristics for neurologic findings at school age is shown in Table IV. When FMs were absent, the concurrent motor repertoire was always abnormal, a combination which was highly predictive for the development of $\mathrm{CP}$. When FMs were abnormal, the concurrent motor repertoire also was always abnormal. This combination preceded the development of complex MND in 7 of 11 infants (64\%). When FMs were normal, an abnormal concurrent motor repertoire preceded the development of complex MND (or worse) in 9 of 28 infants (32\%). Finally, when both FMs and the concurrent motor repertoire were normal, only 1 infant of 21 (5\%) developed complex MND.

Because the different qualitative aspects of the motor repertoire are likely to be interdependent, we performed a multiple logistic regression analysis to investigate which aspects contributed independently to the development of complex MND. Infants who had developed CP were excluded from the analysis, leaving 60 infants, recorded at 11 to 16 weeks, for further analysis. Aspects of the motor repertoire and clinical data that had demonstrated significant associations with later neurologic outcome were entered as predictors: quality of FMs, quality of the concurrent motor repertoire, Apgar score at 5 minutes, and presence of prolonged flaring (PVL grade 1). Only the quality of FMs (likelihood ratio $[\mathrm{LR}]=4.7 ; 95 \%$ confidence interval $[\mathrm{CI}]=0.99$ to 22.5; $P=.05)$, quality of the concurrent motor repertoire $(\mathrm{LR}=14.3 ; 95 \% \mathrm{CI}=1.1$ to $192 ; P=.045)$, and Apgar score at 5 minutes (LR $=0.53 ; 95 \% \mathrm{CI}=0.32$ to $0.87 ; P=$ .012) remained in the model. When we combined the qualitative characteristics of the motor repertoire as a single measure, the LR for development of complex MND was 2.7 ( $95 \%$ $\mathrm{CI}=1.5$ to $4.9 ; P=.001)$.

\section{DISCUSSION}

In children born preterm, the quality of the motor repertoire at 11 to 16 weeks postterm is related to clinically relevant MND (complex MND) at age 7 to 11 years. Both characteristics predictive for an abnormal outcome and characteristics predictive for a normal outcome were identified. Indicative for an abnormal outcome was the presence of abnormal FMs, which was followed by complex MND in nearly $67 \%$ of the cases. Features that were predictive of normal findings at school age were normal FMs in conjunction with a normal concurrent motor repertoire at 11 to 16 weeks. The quality of FMs also differentiated infants who developed complex MND from those who developed CP. FMs were absent at 11 to 16 weeks postterm in most infants who developed CP but not so in those who developed complex MND.

Assessment of the quality of the motor repertoire at this very young age adds considerably to our ability to determine the risk that individual preterm infants will develop clinically relevant, complex MND at school age. Previous studies failed to identify risk factors besides prematurity for the develop- 
Table IV. Association between the combination of quality of FMs and the quality of the concurrent motor repertoire at II to 16 weeks postterm and neurologic findings at school age

\begin{tabular}{|c|c|c|c|c|c|}
\hline \multirow{2}{*}{$\begin{array}{l}\text { Quality of FMs at } \\
\text { I I to I } 6 \text { weeks } \\
\text { postterm }\end{array}$} & \multirow{2}{*}{$\begin{array}{c}\text { Quality of the concurrent motor } \\
\text { repertoire at II to } 16 \text { weeks } \\
\text { postterm }\end{array}$} & \multicolumn{3}{|c|}{ Neurologic findings at school age } & \multirow[b]{2}{*}{ Total } \\
\hline & & $\begin{array}{c}\text { Normal/simple } \\
\text { MND }\end{array}$ & $\begin{array}{l}\text { Complex } \\
\text { MND }\end{array}$ & $\begin{array}{c}\text { Cerebral } \\
\text { palsy }\end{array}$ & \\
\hline \multirow[t]{2}{*}{ Normal } & Smooth and variable & 20 & I & & 21 \\
\hline & Abnormal: monotonous, jerky, and/or stiff & 19 & 8 & 1 & 28 \\
\hline Abnormal & Abnormal: monotonous, jerky, and/or stiff & 4 & 7 & & $\mathrm{II}$ \\
\hline Absent & Abnormal: monotonous, jerky, and/or stiff & & 1 & 12 & 13 \\
\hline
\end{tabular}

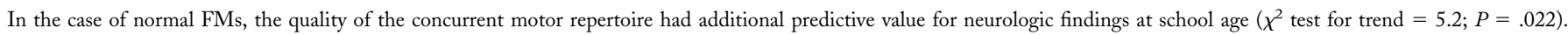

ment of MND. ${ }^{1,2,25}$ In the present study, clinical characteristics from the perinatal period hardly differentiated infants developing complex MND from infants later classified as normal. Only prolonged flaring (PVL grade 1) and a low Apgar score at 5 minutes were associated with development of complex MND. Prolonged flaring was previously associated with minor neurologic signs and perceptual-motor difficulties in preterm infants, ${ }^{26}$ but a recent study did not confirm this association. ${ }^{27}$ Low Apgar score also has been associated with neurologic and developmental disabilities, but its predictive value in the individual infant is very poor. ${ }^{28}$

Our findings extend the results of previous studies that have investigated the predictive value of the quality of the early motor repertoire as a predictor for MND..$^{6-8,29}$ Assessment of muscle power in the shoulders and legs at 3 months corrected age was found to have some predictive value for MND at age 7 years, but risk assessment in the individual infant was low. ${ }^{29}$ Groen et $\mathrm{al}^{8}$ found an association between abnormal GMs and coordination problems and fine motor disabilities at school age; ${ }^{8}$ they did not take the quality of the FMs into account, however. The present study emphasizes the importance of assessing FMs. Together with the quality of the concurrent motor repertoire, the quality of FMs increases the predictive power for MND in the individual infant. Previously, abnormal FMs have been associated with poorer fine motor abilities during midpuberty. ${ }^{30}$ The biological function of FMs as a transient, age-specific, distinct form of GMs remains unclear. It has been speculated that these small movements have an ontogenetic, adaptive function in calibrating the proprioceptive system. ${ }^{31}$ Our findings that abnormal FMs frequently precede clinically relevant abnormalities of coordination, balance, and fine motor abilities at school age lend support to this speculation.

Approximately $50 \%$ of the infants who developed complex MND had normal FMs. There must be another explanation for why these infants developed complex MND. In almost all of the cases, the quality of the concurrent repertoire was abnormal at 11 to 16 weeks. In particular, a monotonous but not a jerky or stiff concurrent motor repertoire was associated with the later development of complex MND. This association was not as strong as that with the presence of abnormal FMs. Several infants with normal FMs in conjunction with an abnormal concurrent repertoire were later classified as normal. However, this combination posed a risk of approximately $30 \%$ for the development of complex MND. Other investigators also have found that although reduced variability of spontaneous motility at this young age increases the risk for later neurologic abnormalities, a slight majority of these infants will develop normally. ${ }^{6,8,32}$ Apparently, reduced variability of the concurrent motor repertoire at this age hampers normal motor development in some, but not all, infants.

The strongest associations between the quality of early movements and the development of complex MND were seen at 11 to 16 weeks postterm. The quality of FMs and of the concurrent repertoire at 6 to 10 and 17 to 24 weeks postterm was less predictive for the development of MND. Previous studies in normally developing children have demonstrated that FMs can be absent before 9 weeks and after 16 weeks postterm age. ${ }^{3}$ This is consistent with the notion that this period can be considered one of major transformation of the nervous system. ${ }^{33}$ During this transformation, many neural functions (eg, postural control, control of visual attention, sucking pattern, social smiling and pleasure vocalizations, motor behavior) change during a relatively short period of several weeks. ${ }^{3,33}$

In the present study, we also were able to identify infants at low risk for developing complex MND. Normal quality of the concurrent motor repertoire at 11 to 16 weeks postterm, in combination with normal FMs, was an excellent marker for a normal neurologic outcome at school age. This is an important observation for distinguishing between infants who need close surveillance and those who do not. It also may help determine whether early intervention (eg, physical therapy) is indicated, especially when resources are limited.

Our findings can be interpreted in at least 2 ways. The first is that normal FMs, in conjunction with a smooth variable concurrent motor repertoire, provide a broad measure of the quality of early central nervous system development. Cortical areas increase their level of activity markedly during the second and third months postterm, as do the cerebellar cortex and the basal ganglia, areas involved in networks with important motor, cognitive, and behavioral functions. ${ }^{34}$ The presence of normal FMs in conjunction with a normal concurrent motor repertoire may be an index of the quality of early central nervous system development in different areas of the brain. On the other hand, a monotonous motor repertoire 
might reflect an abnormal development of the cortical and integrative processes that occur at this age.

A second interpretation is that normal FMs, in conjunction with a smooth variable concurrent motor repertoire, reflect the role that this kind of motor activity plays in the early development of coordination, balance, and fine motor abilities. The infants' exploration of the environment, varying several motor strategies and integrating and refining neural input and output, improves neurologic development. ${ }^{35}$ The absence of a smooth variable motor repertoire at this age might hamper an infant's ability to interact with the environment during a phase in which sensorimotor activity drives motor development.

The present study has some limitations. First, we did not include a term control group, so we do not know how often an abnormal concurrent motor repertoire occurred in low-risk and high-risk term infants. These results cannot be generalized and need to be confirmed in other groups of infants. For this reason, we did not provide data on the sensitivity and specificity of the qualitative assessment of the motor repertoire as described here. A second limitation might be the selection of infants who had participated in several earlier studies that examined the quality of general movements in several groups at risk for neurologic abnormalities. Nonetheless, we consider our study group to be a representative sample of a third-level NICU, because it includes both low-risk and high-risk preterm infants.

We found that several aspects of the quality of the motor repertoire at 11 to 16 weeks postterm were related to complex MND at age 7 to 11 years in children born preterm. Assessment of the quality of the motor repertoire at this age adds considerably to the ability to determine an individual infant's risk for developing clinically relevant complex MND at school age. The results of the present study suggest the following practical approach for identifying whether or not a preterm infant is at risk for later complex MND. First, at 11 to 16 weeks postterm, the assessment of the quality of FMs is important. Abnormal FMs identify those infants at high risk for developing complex MND (60\% to 70\%). Normal FMs necessitate a closer look at the concurrent motor repertoire. If FMs are normal at 11 to 16 weeks postterm, then a smooth, variable concurrent motor repertoire is a marker for a normal outcome, and the risk for developing complex MND is low $(5 \%)$. If the quality of the concurrent motor repertoire is monotonous, then the risk for developing complex MND is approximately $30 \%$.

Our approach outlined herein enables the early identification of individual preterm infants at increased risk for development of MND and thus offers opportunities for early intervention and treatment. Conversely, it also allows for early identification of those preterm infants at very low risk for development of MND.

The authors would like to acknowledge E. F. Stremmelaar, MD, for the support with the clinical follow-up. We are grateful to University of Groningen and the Graduate School for Behavioural and Neuroscience (BCN) for their support.

\section{REFERENCES}

1. van Baar AL, van Wassenaer AG, Briet JM, Dekker FW, Kok JH. Very preterm birth is associated with disabilities in multiple developmental domains. J Pediatr Psychol 2005;30:247-55

2. Bhutta AT, Cleves MA, Casey PH, Cradock MM, Anand KJS. Cognitive and behavioral outcomes of school-aged children who were born preterm: a meta-analysis. JAMA 2002;288:728-37

3. Einspieler C, Bos AF, Ferrari F, Cioni G, Prechtl HFR. Prechtl's Method on the Qualitative Assessment of General Movements in Preterm, Term and Young Infants. London: McKeith Press; 2004.

4. Prechtl HFR, Einspieler C, Cioni G, Bos AF, Ferrari F, Sontheimer D. An early marker for neurological deficits after perinatal brain lesions. Lancet 1997;349:1361-3.

5. Bos AF, Martijn A, van Asperen RM, Hadders-Algra M, Okken A, Prechtl HFR. Qualitative assessment of general movements in high-risk preterm infants with chronic lung disease requiring dexamethasone therapy. J Pediatr 1998;132:300-6.

6. Hadders-Algra M, Mavinkurve-Groothuis AMC, Groen SE, Stremmelaar EF, Martijn A, Butcher PR. Quality of general movements and the development of minor neurological dysfunction at toddler and school age. Clin Rehabil 2004;18:287-99.

7. Hadders-Algra M, Groothuis AMC. Quality of general movements in infancy is related to neurological dysfunction, ADHD, and aggressive behaviour. Dev Med Child Neurol 1999;41:381-91.

8. Groen SE, de Blecourt ACE, Postema K, Hadders-Algra M. General movements in early infancy predict neuromotor development at 9 to 12 years of age. Dev Med Child Neurol 2005;47:731-8

9. Bos AF, Einspieler C, Prechtl HFR, Touwen BCL, Okken-Beukens MD, Stremmelaar EF. The quality of spontaneous motor activity in preterm infants as early predictive signs for minor neurological abnormalities at 2 years. News Neonatal Neurol 1999;8:4-5.

10. Bos AF, van Asperen RM, de Leeuw DM, Prechtl HFR. The influence of septicaemia on spontaneous motility in preterm infants. Early Hum Dev 1997;50:61-70. 11. Bos AF, van Loon AJ, Hadders-Algra M, Martijn A, Okken A, Prechtl HFR. Spontaneous motility in preterm, small for gestational age infants. II: qualitative aspects. Early Hum Dev 1997;50:131-47.

12. Bos AF, Martijn A, Okken A, Prechtl HFR. Quality of general movements in preterm infants with transient periventricular echodensities. Acta Paediatr 1998;87:328-35.

13. Cioni G, Bos AF, Einspieler C, Ferrari F, Martijn A, Paolicelli PB, et al. Early neurological signs in preterm infants with unilateral intraparenchymal echodensity. Neuropediatrics 2000;31:240-51.

14. Butcher PR, Kalverboer AF, Geuze RH, Stremmelaar EF. A longitudinal study of the development of shifts of gaze to a peripheral stimulus in preterm infants with transient periventricular echogenicity. J Exp Child Psychol 2002;82:116-40.

15. Einspieler C, Cioni G, Paolicelli PB, Bos AF, Dressler A, Ferrari F, et al. The early markers for later dyskinetic cerebral palsy are different from those for spastic cerebral palsy. Neuropediatrics 2002;33:73-8.

16. Cioni G, Prechtl HFR. Preterm and early postterm motor behaviour in low-risk premature infants. Early Hum Dev 1990;23:159-91.

17. Dibiasi J, Einspieler C. Can spontaneous movements be modulated by visual and acoustic stimulation in 3-month-old infants? Early Hum Dev 2002;68:27-37.

18. Hagberg B, Hagberg G, Olow I. The changing panorama of cerebral palsy in Sweden 1954-1970, I: analysis of the general changes. Acta Paediatr Scand 1975;64:187-92.

19. Touwen BCL. Examination of the Child with Minor Neurological Dysfunction. London: William Heinemann; 1979.

20. Hadders-Algra M. Two distinct forms of minor neurological dysfunction: perspectives emerging from a review of data of the Groningen Perinatal Project. Dev Med Child Neurol 2002;44:561-71.

21. Smits-Engelsman BCM. Movement Assessment Battery for Children. Lisse, The Netherlands: Swets \& Zeitlinger; 1998.

22. Kloosterman GJ. On intrauterine growth: the significance of prenatal care. Int J Gynaecol Obstet 1970;8:895-912.

23. Papile L, Burstein J, Burstein R, Koffler H. Incidence and evolution of subependymal and intraventricular hemorrhage: a study of infants with birth weights less than $1500 \mathrm{~g}$. J Pediatr 1978;92:529-34.

24. de Vries LS, Eken P, Dubowitz LMS. The spectrum of leukomalacia using cranial ultrasound. Behav Brain Res 1992;49:1-6.

25. Fawke J. Neurological outcomes following preterm birth. Semin Fetal Neonatal Med 2007;12:374-82.

26. Jongmans M, Henderson S, de Vries LS, Dubowitz LMS. Duration of periventricular densities in preterm infants and neurological outcome at 6 years of age. Arch Dis Child 1993;69:9-13.

27. Rademaker KJ, Uiterwaal CSPM, Beek FJA, van Haastert IC, Lieftink AF, Groenendaal F, et al. Neonatal cranial ultrasound versus MRI and neurodevelopmental outcome at school age in children born preterm. Arch Dis Child Fetal Neonatal Ed 2005;90:F489-93. 
28. Nelson KB, Ellenberg JH. Apgar scores as predictors of chronic neurologic disability. Pediatrics 1981;68:36-44.

29. Samsom JF, de Groot L, Bezemer PD, Lafeber HN, Fetter WPF. Muscle power development during the first year of life predicts neuromotor behaviour at 7 years in preterm-born high-risk infants. Early Hum Dev 2002;68:103-18.

30. Einspieler C, Marschik PB, Milioti S, Nakajima Y, Bos AF, Prechtl HF. Are abnormal fidgety movements an early marker for complex minor neurological dysfunction at puberty? Early Hum Dev 2007;83:521-5.

31. Prechtl HFR, Cioni G, Einspieler C, Bos AF, Ferrari F. The role of vision on early motor development: lessons from the blind. Dev Med Child Neurol 2001;43:198-201.
32. Samsom JF, de Groot L, Hopkins B. Muscle power in "high-risk" preterm infants at 12 and 24 weeks corrected age: a measure for early detection. Acta Paediatr 2001;90:1160-6.

33. Prechtl HFR. Continuity of Neural Functions from Prenatal to Postnatal Life. Oxford, UK: Blackwell Scientific; 1984

34. Chugani HT, Phelps ME, Mazziotta JC. Positron emission tomography study of human brain functional development. Ann Neurol 1987;22:487-97.

35. Hadders-Algra M. The neuronal group selection theory: a framework to explain variation in normal motor development. Dev Med Child Neurol 2000; 42:566-72.

\section{Years Ago in The Journal of Pediatrics}

\section{Pediatric use of antibacterial agents}

Kempe CH. J Pediatr 1958;53:19-36

C. Henry Kempe provides in this publication a treatise on roughly 2 dozen antibiotics available as single agents or combination products for the treatment of bacterial infections in children. It takes the form of current reviews of antimicrobial agents: spectrum of activity, dosage and route of administration, adverse effects and toxicities, and major uses by pathogen and clinical syndrome. A modern version also would include the all-important pharmacodynamic properties of an agent; it would not include most products that were "hot" in 1958, because of their toxicities, poor efficacy, or emerged bacterial resistance.

The principles of use espoused well in Kempe's summary, however, are still spot on and are quoted verbatim. "A rational approach for the selection of the proper antimicrobial agent in the treatment of children suffering from bacterial disease requires some basic information and a considerable degree of sales resistance to undue or premature advertising claims. It is suggested that the pediatrician formulate a tentative specific etiologic diagnosis when faced with an illness - usually on the basis of clinical impression — but occasionally by employing the help of bacteriologic study prior to the administration of the antibiotic drug and to give such a drug only when indicated. A good rule in therapy appears to be to give a large enough amount of the drug to which the etiologic microorganism is susceptible, by the proper route and as early as possible in infection, and to continue such treatment for an adequate time to ensure eradication of such infection."

A final historical note on principles of publication and education modeled by Kempe is his disclosures of support: the National Institutes of Health, the Smith Kline and French Laboratories, and the Ross Laboratories.

Sarah S. Long, MD

Section of Infectious Diseases

St. Christopher's Hospital for Children

Philadelphia, Pennsylvania 10.1016/j.jpeds.2008.02.001 
Table III. Association between the quality of the concurrent motor repertoire at postterm age 6 to 10 weeks, II to 16 weeks, and 17 to 24 weeks and neurologic findings at school age

\begin{tabular}{|c|c|c|c|c|c|}
\hline \multirow[b]{2}{*}{ Postterm age, weeks } & \multirow{2}{*}{$\begin{array}{l}\text { Quality of the concurrent } \\
\text { motor repertoire }\end{array}$} & \multicolumn{3}{|c|}{ Neurologic findings at school age } & \multirow[b]{2}{*}{ Total } \\
\hline & & Normal/simple MND & Complex MND & Cerebral palsy & \\
\hline \multirow[t]{3}{*}{6 to 10} & Normal & 13 & 4 & & 17 \\
\hline & Abnormal & 21 & $\mathrm{II}$ & $\mathrm{II}$ & 43 \\
\hline & Total & 34 & 15 & $\mathrm{II}$ & 60 \\
\hline \multirow[t]{3}{*}{11 to 16} & Normal & 20 & 1 & & 21 \\
\hline & Abnormal & 23 & 16 & 13 & 52 \\
\hline & Total & 43 & 17 & 13 & 73 \\
\hline \multirow[t]{3}{*}{17 to 24} & Normal & 15 & 2 & & 17 \\
\hline & Abnormal & 19 & 8 & 9 & 36 \\
\hline & Total & 34 & 10 & 9 & 53 \\
\hline
\end{tabular}

Signs of abnormality of the concurrent motor repertoire include reduced complexity (monotony), jerkiness, and/or stiffness. The numbers in the boxes refer to the number of infants. These associations were significant for all 3 age groups ( 6 to 10 weeks postterm: $\chi^{2}$ test for trend $=12.0, P=.001$; 11 to 16 weeks postterm: $\chi^{2}$ test for trend $=16.3, P<.001$; 17 to 24 weeks postterm: $\chi^{2}$ test for trend $=7.0, P<.01$ ). 